\title{
Der Zusammenhang zwischen COVID-19 und Schlaganfall: Pathophysiologie und klinische Bedeutung
}

\section{Association of COVID-19 and Stroke: Pathophysiology and Clinical Relevance}

\author{
Autoren \\ Timo Siepmann ${ }^{1}$, Kristian Barlinn ${ }^{1}$ \\ Institute \\ 1 Klinik und Poliklinik für Neurologie, Universitätsklinik Carl \\ Gustav Carus, Technische Universität Dresden
}

Schlüsselwörter

COVID-19, SARS-CoV-2, Schlaganfall, Hyperkoagulabilität, Vaskulitis, Kardiomyopathie

Keywords

COVID-19, SARS-CoV-2, Stroke, Hypercoagulability, Vasculitis, Cardiomyopathy

eingereicht $\quad 09.02 .2021$

akzeptiert $\quad 08.04 .2021$

Bibliografie

Fortschr Neurol Psychiatr 2021; 89: 289-295

DOI 10.1055/a-1484-0224

ISSN 0720-4299

(C) 2021. Thieme. All rights reserved.

Georg Thieme Verlag KG, Rüdigerstraße 14,

70469 Stuttgart, Germany

Korrespondenzadresse

PD Dr. med. habil. Timo Siepmann

Klinik und Poliklinik für Neurologie, Universitätsklinik Carl

Gustav Carus, Technische Universität Dresden

Fetscherstr. 74

01307 Dresden

Deutschland

Tel.: +49-351-458-3503

Fax: + 49-351-458-4365

E-Mail: Timo.Siepmann@ukdd.de

\section{ZUSAMMENFASSUNG}

Dass COVID-19 mit einem erhöhten Risiko für das Auftreten ischämischer Schlaganfälle einhergeht, wurde bereits in den ersten Monaten nach dem weltweiten Bekanntwerden des Ausbruchs der Pandemie im Ground Zero Wuhan deutlich. Spätere Kohortenanalysen, Fallserien und Meta-Analysen bestätigten diesen Zusammenhang. Eine nicht unerhebliche geographische Variabilität der Prävalenz COVID-19-bezogener Schlaganfälle verdeutlicht allerdings den fortbestehenden Bedarf an umfassenden epidemiologischen Analysen. Im Übrigen fanden sich zunehmend Hinweise dafür, dass dem mit COVID-19 assoziierten Schlaganfall ein multifaktorieller pathophysiologischer Mechanismus zugrundeliegenden könnte. Neben einer durch die SARS-CoV-2-Infektion verursachten Hyperkoagubilität, die sowohl im mikrovaskulären System als auch in Großgefäßen thrombogen wirkt, scheinen vaskulitische arterielle Veränderungen und kardiogene Embolien infolge virusassoziierter myokardialer Schädigung bei der Ätiologie des COVID-19-assoziierten Schlaganfalls eine Rolle zu spielen. Die Versorgung von Schlaganfallpatienten/innen in Zeiten der Pandemie durch multidisziplinäre Teams scheint in insgesamt kompensierten Kliniken im Wesentlichen nicht kompromittiert zu sein. Hinweise, dass Patienten/innen mit COVID-19-assoziiertem Schlaganfall von einem intensivierten Monitoring und einer aggressiveren Sekundärprävention profitieren, bedarf klinischer Prüfung.

\section{ABSTRACT}

The notion of an increased risk of acute ischemic stroke in patients with COVID-19 emerged during the first months after the outbreak of the COVID-19 pandemic at ground zero in Wuhan. Subsequently, this association was confirmed by multiple case reports, cohort studies and meta-analyses. However, substantial geographical variability in prevalence of COVID-19 associated stroke underscores a persistent need of encompassing epidemiological study. Accumulative evidence suggested a rather complex underlying pathophysiology. Possible mechanisms include small and large vessel thromboembolism induced by systemic hypercoagubility, vasculitis and cardiac embolism caused by virus-induced cardiomyopathy. Multidisciplinary care of acute stroke patients in general does not seem to be compromised by the pandemic in otherwise compensated hospitals. However, it remains to be answered whether more aggressive approaches of secondary prevention and intensified monitoring strategies after COVID-19 related stroke are necessary to optimize clinical outcomes. 
Klinische Evidenz zur durch COVID-19 bedingten Risikoerhöhung für das Auftreten eines Schlaganfalls

Bereits in den ersten Monaten der Ausbreitung des Severe Acute Respiratory Syndrome Coronavirus 2 (SARS-CoV-2) in der auch als „Ground Zero“ bezeichneten Unterprovinzstadt der Chinesischen Provinz Hubei, Wuhan, wurde deutlich, dass die aus einer Infektion mit dem Virus resultierende Erkrankung Coronavirus Disease 2019 (COVID-19) nicht auf das respiratorische System beschränkt ist. Eine rasch steigende Anzahl an Fallberichten und retrospektiven Analysen von COVID-19-Patienten/innen, die eine nicht anderweitig erklärbare Störung des Geruchs- und/oder des Geschmackssinns erlitten, sowie eine erhöhte Inzidenz epileptischer Anfälle mit SARS-CoV-2 infizierten Patienten/innen deuteten in dieser frühen Phase der Pandemie bereits darauf hin, dass das Virus die funktionelle Integrität von Bestandteilen des Nervensystems kompromittiert [1, 2]. Parallel wuchs die Anzahl der Fallberichte und Kohortenbeschreibungen, die einen Zusammenhang zwischen einer COVID-19-Erkrankung und thrombembolischen Ereignissen nahelegten [3, 4].

Obgleich eingangs Lungenarterienembolien und venöse Thrombosen im Kontext der COVID-19-Erkrankung im Fokus der Wissenschaft standen, zeigten bereits die frühen Kohorten aus Wuhan sowie die Daten aus der Zeit des norditalienischen Ausbruchs im Frühjahr 2020 eine erhöhte Schlaganfallinzidenz bei SARS-CoV-2-positiven Patienten/innen [1, 5]. In der sich daran anschließenden Zeit wuchs die Anzahl COVID-19-bezogener wissenschaftlicher Publikationen rasant, und im Zuge dieser Entwicklung wurden in zunehmender Quantität auch Kohortenanalysen und Fallserien zum Auftreten von Schlaganfällen bei mit SARS-CoV-2 infizierten Patienten/innen veröffentlicht. Eine rezente systematische Meta-Analyse identifizierte 2628 Artikel, von denen sich 150 zur Volltextanalyse qualifizierten und Daten von insgesamt 3306 Patienten/innen aus 10 Studien lieferten, die in ausreichendem Umfang über den Anteil der COVID19-Patienten/innen berichteten, die einen Schlaganfall erlitten [6]. In dieser gepoolten Population erlitten 1,8\% der COVID-19Patienten/innen einen Schlaganfall, wobei es sich in der Mehrheit um ischämische Schlaganfälle (1,5\% der Gesamtpopulation) handelte. Die Arbeit demonstrierte überdies, dass COVID-19-Patienten/innen, die einen Schlaganfall erleiden, eine stark erhöhte Mortalität von über 34\% aufweisen und dass nahezu die Hälfte aller COVID-19-assoziierter Schlaganfälle auf einen Großgefäßverschluss zurückzuführen ist. Eine andere Meta-Analyse, die Daten zu Kohorten aus Wuhan, Norditalien und Sachsen synthetisierte, konnte darüber hinaus zeigen, dass das Schlaganfallrisiko mit dem Schweregrad der COVID-19-Erkrankung zunimmt und bei schweren Verläufen bis zu 5,5\% beträgt [7]. Ein erhöhtes Schlaganfallrisiko bei schwer erkrankten COVID-19-Patienten/ innen fand sich dabei, unabhängig davon, ob die Erkrankungsschwere durch klinische Verlaufsparameter, die Notwendigkeit intensivmedizinischer Therapie oder durch ein kombiniertes Outcome definiert wurde, welches sämtliche von den Autoren der extrahierten Arbeiten festgelegten Schwerekriterien subsumierte. Eine weitere Meta-Analyse mit gepoolten Daten von insgesamt 55176 COVID-19-Patienten/innen, von denen 899 einen
Schlaganfall erlitten, bestätigte den Zusammenhang zwischen einem weiteren Anstieg des Schlaganfallrisikos mit dem Alter und der Schwere der COVID-19-Erkrankung [8].

Bei der empirischen Betrachtung des Zusammenhangs zwischen COVID-19 und Schlaganfall ist es erwähnenswert, dass ein vorangegangener Schlaganfall auch ein erhöhtes Risiko dafür darstellt, dass eine COVID-Erkrankung schwer verläuft. Eine gepoolte Analyse, die Daten von 1906 laborchemisch bestätigten SARSCoV-2-positiven Patienten/innen enthielt, zeigte, dass solche COVID-19-Patienten/innen, die eine Schlaganfallanamnese aufweisen, ein erhöhtes Risiko haben, einen schweren Verlauf der Viruserkrankung zu erleiden, intensivmedizinische Behandlung zu benötigen oder während des Krankenhausaufenthalts zu versterben [9]. Zunächst verblieb es dabei ungeklärt, ob der beobachtete Zusammenhang darauf zurückzuführen ist, dass Schlaganfallpatienten/innen insgesamt ein kardiovaskuläres Risikoprofil aufweisen, welches sich in der Gesamtheit ungünstig auf den Krankheitsverlauf auswirkt. Spätere Arbeiten konnten aber nachweisen, dass es sich um einen darüber hinaus gehenden schlaganfallspezifischen Effekt handelt. So demonstrierte beispielsweise eine für Komorbiditäten adjustierte Analyse einer großen Kohorte SARS-CoV-2-positiver Patienten/innen aus New York ( $n=3248$ ) eine unabhängige Risikoerhöhung für Mortalität während der COVID-19-bezogenen Hospitalisierung [10]. Überdies zeigte eine rezente vergleichende Kohortenstudie, dass COVID19-Patienten/innen mit zerebralem Großgefäßverschluss nicht nur klinisch, sondern auch bildgebend eine erhöhte Schlaganfallschwere als SARS-CoV-2-negative Patienten/innen aufweisen, die sich in einem größeren Infarktkern und einem geringeren Diffusion-Weighted Imaging-Alberta Stroke Program Early CT Score darstellt [11].

Die Datenlage zur Risikoassoziation von COVID-19 und Schlaganfall ist keinesfalls eindeutig. So konnte eine große transversale Studie (24808 Patienten/innen, davon 566 mit COVID-19) zwar ebenfalls ein ungünstigeres klinisches Outcome bei SARS-CoV-2positiven Schlaganfallpatienten/innen feststellen; ein erhöhtes Schlaganfallrisiko fand sich aber nicht in der COVID-19-Population im Vergleich mit SARS-CoV-2-negativen Patienten/innen [12]. Eine retrospektive Kohortenanalyse von 1916 bestätigten SARS-CoV-2-positiven Patienten/innen und 1486 Patienten/ innen, die an einer laborchemisch bestätigten Influenzainfektion litten, erbrachte ein zu dieser Arbeit konträres Ergebnis. Im Vergleich mit den Influenzapatienten/innen zeigten die COVID-19Patienten/innen 7,6-fach erhöhte Odds, einen Schlaganfall zu erleiden [13]. Weitere Analysen zeigten zwar eine deutliche Variabilität der Schlaganfallhäufigkeit bei COVID-19-Erkrankten mit Inzidenzen zwischen 1 und $6 \%$; konsistent aber zeichnete sich ab, dass die durch SARS-CoV-2 verursachte Viruserkrankung das Risiko für einen Schlaganfall darstellt, welches sich nicht durch die allgemeine Krankheitsschwere allein erklärt, sondern einen COVID-19-spezifischen Befund darstellt [14-17]. Eine Analyse des 28 Studienzentren aus 16 Ländern umfassenden Global Stroke Registry bestätigte, dass COVID-19-Patienten/innen, die einen Schlaganfall erleiden, eine höhere Wahrscheinlichkeit aufweisen, schwere funktionelle Beeinträchtigungen zu entwickeln oder zu versterben als SARS-CoV-2-negative Schlaganfallpatienten/ innen [15]. 
Bei der Interpretation der bisherigen Literatur ist zu berücksichtigen, dass die beobachteten Prävalenzen des COVID-19-assoziierten Schlaganfalls eine erhebliche geographische Variabilität aufweisen, mit bis zu dreifach erhöhter Häufigkeit in asiatischen Populationen verglichen mit europäischen und nordamerikanischen Populationen. Dies könnte in Teilen durch einen Publikationsbias verursacht sein, sodass weitere umfassendere Meta-Analysen und prospektive multizentrische COVID-19Schlaganfallregister trotz der insgesamt sehr hohen Quantität an COVID-19-bezogenen Publikationen dringend benötigt werden.

Zudem unterscheiden sich COVID-19-assoziierte Schlaganfälle phänotypisch von denen, die bei nicht infizierten Patienten/innen auftreten. In einer gepoolten Analyse, die Daten von über hunderttausend (108571) Patienten/innen inkludierte, waren an COVID-19 erkrankte Patienten/innen jünger (gepoolte Mittelwertdifferenz: -6 Jahre), wiesen schwerere neurologische Defizite auf (gepoolte Mittelwertdifferenz des National Institutes of Health Stroke Scale Scores: 5) und hatten eine höhere Wahrscheinlichkeit, im Krankenhaus zu versterben [18]. Von pathophysiologischer Relevanz ist ein weiterer Befund der Arbeit: COVID-19-assoziierte Schlaganfälle werden häufiger durch einen Großgefäßverschluss verursacht als Schlaganfälle bei SARS-CoV-2-negativen Patienten/ innen. Die Emboliequelle wird dabei in vielen Fällen nicht identifiziert, sodass in bis zu 65,6\% der SARS-CoV-2-positiven Schlaganfallpatienten/innen eine kryptogene Ätiologie dokumentiert wird [16]. Dieser Befund ist möglicherweise mit dem auch insgesamt erhöhten Thrombembolierisiko durch COVID-19 vereinbar, welches hinsichtlich der möglicherweise zugrundeliegenden Pathophysiologie und der klinischen Implikationen für die Behandlung von Schlaganfallpatienten/innen in den folgenden Kapiteln näher beleuchtet wird.

\section{Spektrum möglicher pathophysiologischer Mechanismen eines Schlaganfalls bei COVID- 19-Patienten/innen}

Da embolisch konfigurierte Schlaganfälle ungeklärter Ätiologie bei COVID-19-Patienten/innen häufiger vorkommen als bei SARSCoV-2-negativen Personen, liegt es nahe, dass ein erkrankungsspezifischer Mechanismus für die Thrombusbildung ursächlich sein könnte [17]. In der Literatur postulierte und diskutierte pathophysiologische Prozesse umfassen dabei insbesondere die Hyperkoagulabilität-bedingte Thrombenbildung, der vaskulitische Schlaganfall und die SARS-CoV-2-vermittelte Kardiomyopathie. Hämorrhagische Schlaganfälle wurden im Übrigen in geringerem Umfang ebenfalls mit COVID-19 in einen möglichen pathomechanistischen Zusammenhang gebracht, wobei wandarterielle Schäden cerebraler Gefäße durch eine ACE-2-Rezeptor-vermittelte direkte SARS-CoV-2-Virusinvasion in das Endothel diskutiert mit nachfolgender Gefäßruptur sowie eine Cytokin-vermittelte Kompromittierung der strukurellen Integrität der Blut-HirnSchranke als mögliche Ursachen diskutiert wurden [19, 20]. Mögliche pathophysiologische Mechanismen COVID-19-assoziierter Schlaganfälle sind in $>$ Abb. 1 illustriert und im Folgenden im Detail erörtert.

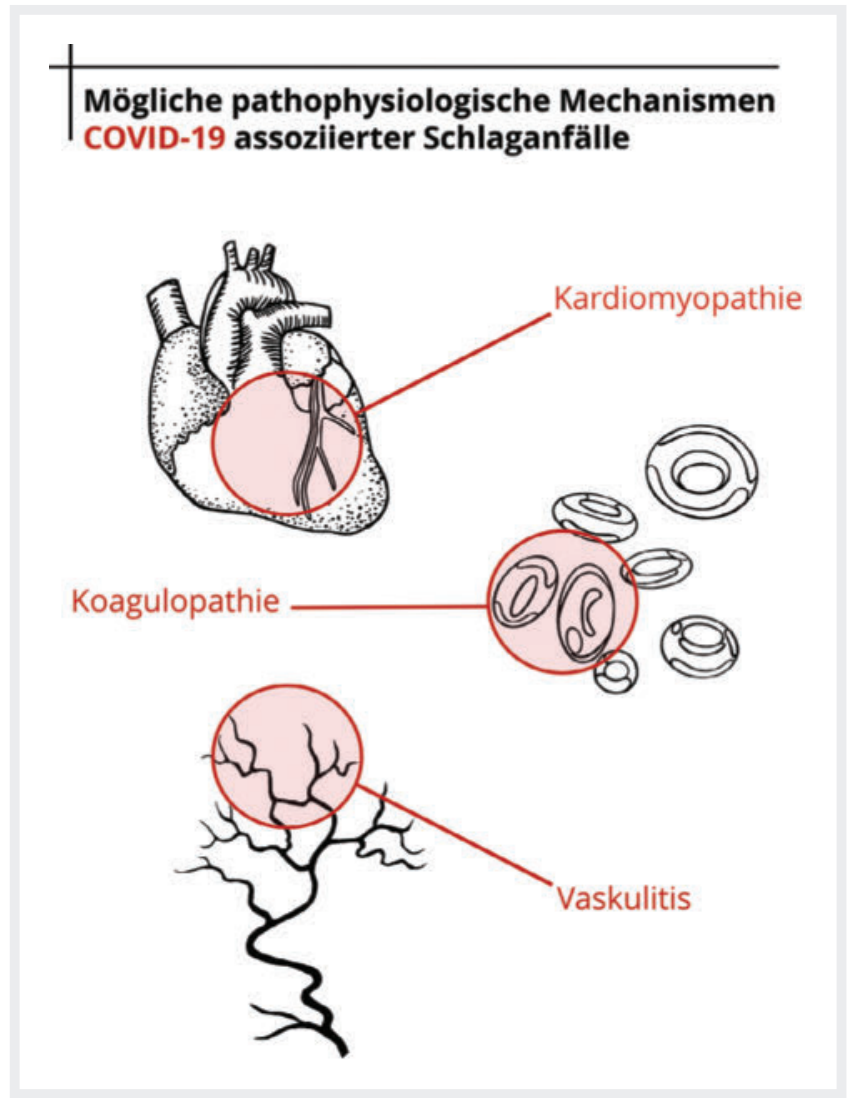

- Abb 1 Illustration der bislang postulierten möglichen pathophysiologischen Mechanismen COVID-19-assoziierter Schlaganfälle. Unabhängig von diesen pathophysiologischen Hypothesen sollten SARS-CoV-2-positive Schlaganfallpatienten/innen die allgemein empfohlene Diagnostik (u. a. zerebrale Bildgebung, Neurosonographie, kardiale Diagnostik, EKG inkl. Langzeit-EKG-Monitoring) und, bei bestehender Indikation, Akuttherapie (intravenöse Thrombolyse und/oder Thrombektomie) erhalten [58]. Eine spezifische Ätiologiediagnostik unter interdisziplinärer Miteinbeziehung kardiologischer, hämastesologischer und angiologischer Fachgebiete sollte je nach bestehender Indizienlage in Bezug auf den vermuteten Pathomechanismus erfolgen. Angesichts der unklaren diesbezüglichen Datenlage ist die individuelle und interdisziplinäre Einschätzung des jeweiligen Falls anzuraten.

\section{Hyperkoagulabilität}

Bei bis zu 55\% der COVID-19-Patienten/innen finden sich laborchemische Hinweise auf eine Koagulopathie, wie erhöhte D-Dimere, eine verlängerte Prothrombinzeit und eine leichte Thrombozytopenie sowie in späteren Erkrankungsstadien auch eine Erhöhung der Fibrinogenkonzentration [21]. Einzelfallberichte von COVID-19-Patienten/innen mit thrombembolischen Ereignissen, wie multiplen embolischen Schlaganfällen und gleichzeitigen Erhöhungen der gegen Cardiolipin und $\beta$-Glycoprotein gerichteten Antiphospholipid Antikörper, begründeten die Hypothese eines mit der COVID-19-Koagulopathie assoziierten Antiphospholipid-Antikörper-Syndroms, die sich in späteren Fallserien SARS-CoV-2-positiver Schlaganfallpatienten/innen zunächst nicht konklusiv bestätigte [22-24]. Wurden laborchemische Analysen allerdings nur in solchen COVID-19-Patienten/ 
innen durchgeführt, die einen schweren Erkrankungsverlauf aufwiesen und intensivmedizinisch behandelt wurden, fanden sich Antiphospholipid-Antikörper auch in bis zu 80\% der Fälle [25]. Interessanterweise schien eine aggressive Antikoagulation das klinische Outcome dieser Patienten/innen zu verbessern.

Während die pathophysiologische Rolle der AntiphospholipidAntikörper bei COVID-19 bislang nicht hinreichend geklärt wurde, fanden sich in einer rasch zunehmenden Anzahl von Kohortenanalysen Indizien für die Existenz einer COVID-19-Koagulopathie, die durch eine systemische Aktivierung des Gerinnungssystems mit assoziierter Schädigung der Mikrozirkulation gekennzeichnet ist [26]. Angesichts eines erhöhten Risikos für thrombotische und thrombembolische Ereignisse hatte die International Society for Thrombosis and Haemostasis bereits im April 2020 ein laborchemisches Monitorung dieser Parameter bei jedem an COVID-19 erkrankten Patienten/innen empfohlen [27]. Klinische Fallserien und Post-mortem-Analysen konnten in der Folge zeigen, dass COVID-19-assoziierte Gerinnungsstörungen sowohl zu thrombotischen Ereignissen der Mikrozirkulation als auch zu Großgefäßverschlüssen in der Lunge, dem Gehirn und anderen Organen führen können [28-30]. Beim COVID-19-assoziierten Schlaganfall sind Großgefäßverschlüsse häufiger als bei SARS-CoV-2-negativen Patienten/innen [15]. Da Patienten/innen mit COVID-19-assoziiertem Schlaganfall insgesamt jünger und weniger schwerer kardiovaskulär vorerkrankt zu sein scheinen als SARS-CoV-2-negative Schlaganfallpatienten/innen, ließe sich möglicherweise schlussfolgern, dass kardioembolische oder paradox-embolische Mechanismen häufiger sind als Thromben, die sich in sich an rupturierten arteriellen Plaques bilden [18]. Damit vereinbar wäre auch die hohe Prävalenz an tiefen Venenthrombosen, die bei bis zu einem Viertel der COVID-19-Patienten/innen auftreten, die eine intensivmedizinische Behandlung benötigen $[31,32]$.

\section{Vaskulitis}

Die Affinität von SARS-CoV-2 für den Angiotensin Converting Enzyme 2 (ACE 2) Rezeptor könnte den bei Patienten/innen mit COVID-19-assoziierter Koagulopathie beobachteten Befund einer lymphozytären Endothelitis erklären, da der Rezeptor nicht nur in Lunge, Herz, Nieren und Intestinum, sondern auch im arteriellen Gefäßendothele exprimiert ist [33]. Die rezeptorvermittelte Virusinvasion auf Zellebene könnte daher ein Bestandteil einer Pathophysiologie COVID-19-assoziierter Koagulopathie darstellen, die neben der Funktion des Gerinnungssystems auch die endotheliale Struktur des Gefäßsystems attackiert und zur Thrombemboliebildung sowie zu hämodynamischen Ischämien beiträgt [34]. Angesichts des insbesondere bei schwer betroffenen COVID-19Patienten/innen häufig beobachteten, u.a. durch die Proteine Interleukin 1B, Interferon $\mathrm{y}$, Interferon-gamma Induced Protein 10 kD und Makrophagen-Chemoattraktorprotein-1 vermittelten „Cytokinsturms“ wäre es auch denkbar, dass vaskulitische Veränderungen nicht nur eine Folge der ACE 2 Rezeptor-vermittelten Endothelinvasion darstellen, sondern überdies durch eine systemische Störung der Mikrozirkulation begünstigt wird, die bei betroffenen Patienten/innen auch zur Multi-Organ-Dysfunktion beiträgt [32, 35].
Die Hypothese einer vaskulitischen Komponente der Pathophysiologie COVID-19-assoziierter Schlaganfälle wäre auch konsistent mit häufig beobachteten extracerebralen Vaskulitismanifestationen bei COVID-19-Patienten/innen, wie der kutanen Vaskulitis oder der kardialen (koronararteriellen) Vaskulitis [36]. Die Datenlage im Hinblick auf zerebrale und/oder zervikale SARS-CoV-2-assoziierte Vaskulitiden und deren Anteil der erhöhten Schlaganfallinzidenz bei COVID-19-Patienten/innen ist äußerst heterogen. Insbesondere ist bei der Beurteilung dieser Hypothese zu berücksichtigen, dass zwar eine schwere Entzündungsreaktion stattfindet, die auch zu Endothelschäden führt. Diese scheint aber auch auf das Endothel begrenzt zu sein, anders als bei einer Herpes-Vaskulitis [37]. Gegen die Vaskulitishypothese sprechen auch die oftmals beobachteten embolischen Muster COVID-19-assoziierter Schlaganfälle.

\section{Kardiomyopathie}

Strukturelle Schädigungen des Herzmuskels kommen in bis zu $30 \%$ der Patienten/innen mit COVID-19 vor [37]. Verschiedene Mechanismen einer SARS-CoV-2-vermittelten Kardiomyopathie wurden diskutiert. Neben einer myokardialen Virusinvasion auf zellulärer Ebene mit konsekutiver lokaler Entzündungsreaktion finden sich auch in zunehmendem Umfang Hinweise für eine durch respiratorische Insuffizienz bedingte hypoxische Herzschädigung sowie eine sekundäre kardiale Inflammation infolge SARS-CoV-2-assoziierter systemischer Inflammation in der Literatur, wobei die myokardiale Inflammation in mehr als der Hälfte der betroffenen COVID-19-Patienten/innen auch nach der klinischen Remission der Erkrankung und Erreichen einer negativen SARS-CoV-2-PCR noch anzuhalten scheint [38]. Die mit einer SARS-CoV-2 assoziierte strukturelle myokardiale Schädigung stellt eine potenzielle Ursache für Arrhythmien und lokaler Strömungsalterationen dar und könnte daher das erhöhte Schlaganfallrisiko bei COVID-19-Patienten/innen und insbesondere auch den erhöhten Anteil an embolischen Großgefäßverschlüssen in dieser Population erklären. Ein erhöhtes Risiko für kardiale Arrhythmien könnte sich auch durch die mit der akuten Viruserkrankung und der auch als Cytokinsturm bezeichneten ausgeprägten Immunreaktion einhergehenden Aktivierung des sympathischen Nervensystems begründen, welche einerseits durch Reduktion der Herzfrequenzvariabilität und andererseits durch eine Tachykardisierung arrhythmogen wirken kann $[39,40]$.

\section{Klinisches Management des Schlaganfalls in der Pandemie und Besonderheiten der Behandlung des COVID-19-Schlaganfalls}

In den Monaten nach Beginn der SARS-CoV-2-Pandemie wurde eine Reduktion der Anzahl aufgrund eines Schlaganfalls oder einer transitorischen ischämischen Attacke hospitalisierten Patienten/innen beobachtet [41]. Die Pandemie schien dazu zu führen, dass Patienten/innen seltener das medizinische Notversorgungssystem in Anspruch nahmen, möglicherweise aufgrund der Infektionsgefahr. Schlaganfallpatienten/innen erhielten überdies weniger wahrscheinlich eine Akuttherapie via 
endovaskulärer Thrombektomie oder intravenöser Lystherapie, als dies ein Jahr zuvor, also vor dem COVID-Ausbruch, noch der Fall war [42]. Obwohl sich zunächst Hinweise dafür fanden, dass die Versorgungsqualität der behandelten Schlaganfallpatienten/ innen nicht unter der Pandemie leidet, erregte die Entwicklung angesichts der Möglichkeit einer drastischen Zunahme unbehandelter Schlaganfälle Besorgnis in der internationalen SchlaganfallCommunity und führte zu der Forderung, die Schlaganfallversorgung während der SARS-CoV-2-Pandemie standardisiert zu erfassen und gezielt und flächendeckend zu verbessern [43, 44].

Maßgeschneiderte prähospitale Screeningkonzepte und multidisziplinäre „Protected Code Stroke“-Protokolle wurden entwickelt, um die akute Schlaganfalltherapie ohne Einbußen in der Behandlungseffektivität, der Geschwindigkeit oder dem Infektionsschutz zu tolerieren [45]. Weitere Analysen zeichneten auch hinsichtlich der individuellen Behandlungsqualität ein heterogenes Bild. Beispielsweise war den Resultaten einer retrospektiven multizentrischen Analyse zufolge, die Onset-to-groin puncture time, die für das neurologische Outcome relevante Dauer zwischen dem Symptomeinsatz und der Leistenarterienpunktion im Rahmen der endovaskulären Behandlung fast eine ganze Stunde (54 Minuten) verlängert im Vergleich zur Prä-COVID-Ära [46]. Eine multizentrische Studie aus Deutschland zeigte wiederum keine Unterschiede in der endovaskulären Therapie und den assoziierten klinischen Outcomes während der Pandemie im Vergleich mit der Prä-COVID-Ära [47]. Eine prospektive monozentrische Beobachtungsstudie, welche im Zuge der ersten Infektionswelle durchgeführt wurde, zeigte keinen nachteiligen Einfluss eines spezifischen Infektionsprotokolls mit routinemäßiger laborchemischer Testung auf SARS-CoV-2 auf die Door-toimaging-Zeit, einer weiteren relevanten Prozedurzeit zwischen Eintreffen in die Notaufnahme und dem Zeitpunkt der Akutbildgebung bei Patienten, die eine intravenöse Lysetherapie oder endovaskuläre Therapie erhielten [48]. Eine retrospektive monozentrische Kohortenanalyse, stellte fest, dass die Pandemie zwar die Rate an rekanalisierenden Therapien nicht durch COVID19 kompromitiert wurde, es aber weniger Krankenhausvorstellungen wegen transitorischer ischämischer Attacken und Schlaganfällen kam, sodass ein besonderer Fokus der Aufmerksamkeit im Bereich der transienten und milden neurologischen Symptome sinnvoll sein könnte [49].

Die vorliegende Literatur unterstreicht die Notwendigkeit einer Standardisierung und Integration schlaganfallspezifischer Behandlungsalgorithmen in den Kontext des Infektionsschutzes. Maßnahmen, die sich als effektiv erweisen könnten, umfassen neben dem labormedizinischen Untersuchung aller Schlaganfallpatienten/innen hinsichtlich des Vorliegens einer SARS-CoV-2-Infektion auch den vermehrten Einsatz telemedizinischer Schlaganfallbehandlung und mobiler Stroke Units sowie die intensivierte Aufklärung der Bevölkerung über die Erkennung akuter Schlaganfallsymptome im Rahmen von gezielten AwarenessKampagnen [50, 51].

Angesichts des bei COVID-19-Patienten/innen erhöhten Thrombembolierisikos und des vermutlich damit zumindest partiell in Kausalzusammenhang stehenden erhöhten Schlaganfallrisikos ergibt sich die Frage, wie COVID-19-Patienten/innen, die einen Schlaganfall erleiden, optimal vor einer thrombotischen oder thrombembolischen Komplikation durch effektive Sekundärprävention geschützt werden können und ob dies eine aggressivere Antikoagulation erfordert, als es bei SARS-CoV-2negativen Schlaganfallpatienten/innen erforderlich ist. Dies ist insbesondere vor dem Hintergrund relevant, dass Schlaganfallpatienten/innen unabhängig von COVID-19 ein stark erhöhtes Thromboserisiko haben, mit Wahrscheinlichkeiten von bis zu $50 \%$, eine venöse Thrombose zu erleiden, und dass SARS-CoV-2positive Schlaganfallpatienten/innen, die bereits eine medikamentöse Thromboseprophylaxe erhalten, in bis zu 4,5\% der Fälle eine Thrombose trotz Heparintherapie erleiden [52-54]. Bei COVID-19-Patienten, die eine ausgeprägte Erhöhung der D-Dimere aufweisen, und bei solchen, die eine intensivmedizinische Behandlung bedürfen, fanden sich erste Hinweise dafür, dass die therapeutische Antikoagulation anstelle der üblichen Thromboseprophylaxe mit Low Dose Heparin das klinische Outcome verbessern [54-56]. Die Rekrutierung schwer erkrankter COVID-19Patienten/innen im Rahmen von 3 konfirmatorischen randomisierten kontrollierten Phase-III-Studien zur prophylaktischen Vollantikoagulation (the Randomized, Embedded, Multi-factorial Adaptive Platform Trial for Community-Acquired Pneumonia [REMAP-CAP]; Accelerating COVID-19 Therapeutic Interventions and Vaccines-4 [ACTIV-4]; Antithrombotic Therapy to Ameliorate Complications of COVID-19 [ATTACC]) wurden jedoch im Dezember 2020 aufgrund eines von den Oversight Boards festgestellten Verfehlens einer Verbesserung des klinischen Outcomes (Reduktion der Notwendigkeit organersetzender Therapie) bei gleichzeitigen Sicherheitsbedenken gestoppt [57]. Leichte und moderate COVID-19-Fälle sind jedoch nicht Gegenstand des Rekrutierungsstopps. Insgesamt besteht eine erhebliche Unsicherheit bezüglich der optimalen Prophylaxe thrombembolischer Ereignisse bei COVID-19-Patienten/innen. Inwieweit sich der aktuelle $\mathrm{Er}$ kenntnisstand auf die Behandlung SARS-CoV-2-positiver Schlaganfallpatienten/innen übertragen lässt, verbleibt im Wesentlichen ungeklärt und bedarf dringend einer klinischen Prüfung in relevanten Populationen SARS-CoV-2-positiver Schlaganfallpatienten/innen.

Aus aktueller Sicht sind die konsequente Thromboseprophylaxe, nicht pharmakologische präventive Maßnahmen, wie Mobilisierung und mechanische Thromboseprophylaxe, die sorgfältigen individuellen Risikoevaluation und das konsequente Monitoring laborchemischer Gerinnungsparameter bei infizierten Schlaganfallpatienten/innen zu empfehlen. Empfehlungen zur Schlaganfallversorgung im Kontext von COVID-19 finden sich in der Leitlinie der Deutschen Gesellschaft für Neurologie [58].

\section{Schlussfolgerungen}

Der COVID-19-assoziierte Schlaganfall scheint sich nach gegenwärtigem Wissensstand sowohl hinsichtlich der zugrundeliegenden Pathophysiologie als auch in Bezug auf klinische Outcomes von Schlaganfällen bei SARS-CoV-2-negativen Patienten/innen zu unterscheiden. Daraus ergeben sich Herausforderungen für die diagnostischen und therapeutischen Versorgungsabläufe, das schlaganfallbezogene Monitoring und die Sekundärprävention, die einer klinischen Prüfung in großen Patientenpopulation bedürfen, um eine flächendeckende 
optimale Versorgung des COVID-19-Schlaganfalls zu ermöglichen. Zugleich ergibt sich ein dringender Bedarf an umfassenden Analysen der Epidemiologie und der zugrundeliegenden Mechanismen des COVID-19-assoziierten Schlaganfalls, um die Entwicklung individualisierter präventiver und therapeutischer Konzepte zu ermöglichen.

\section{Interessenkonflikt}

PD Dr. med. habil. Timo Siepmann erhielt Forschungsfördermittel von dem Bundesministerium für Gesundheit, dem Kurt Goldstein Institut, der Michael J. Fox Foundation, der Deutschen Parkinsongesellschaft, die nicht mit dem vorliegenden Artikel in Verbindung stehen. Er erhielt Honorare von Thieme als Herausgeber des Lehrbuchs „Arzneimitteltherapie“, von der Dresden International University für seine Tätigkeit als Wissenschaftlicher Leiter des Masterstudiengangs „Clinical Research“ und von Astrazeneca für Beratungsleistungen.

\section{Literatur}

[1] Mao L, Jin $\mathrm{H}$, Wang $\mathrm{M}$ et al. Neurologic manifestations of hospitalized patients with coronavirus disease 2019 in Wuhan, China. JAMA Neurol 2020; 77 (6): 683-690. doi: 10.1001/jamaneurol.2020.1127

[2] Niazkar HR, Zibaee B, Nasimi A et al. The neurological manifestations of COVID-19: A review article. Neurol Sci 2020; 41 (7): 1667-1671. 10.1007/s10072-020-04486-3

[3] Bikdeli B, Madhavan MV, Jimenez D et al. COVID-19 and thrombotic or thromboembolic disease: implications for prevention, antithrombotic therapy, and follow-up: JACC state-of-the-art review. J Am Coll Cardiol 2020; 75 (23): 2950-2973. doi: 10.1016/j.jacc.2020.04.031

[4] Cui S, Chen S, Li X et al. Prevalence of venous thromboembolism in patients with severe novel coronavirus pneumonia. J Thromb Haemost 2020; 18 (6): 1421-1424. 10.1111/jth.14830

[5] Lodigiani C, lapichino G, Carenzo L et al. Venous and arterial thromboembolic complications in COVID-19 patients admitted to an academic hospital in Milan, Italy. Thromb Res 2020; 191: 9-14. 10.1016/j. thromres.2020.04.024

[6] Fridman S, Bres Bullrich M, Jimenez-Ruiz A et al. Stroke risk, phenotypes, and death in COVID-19: systematic review and newly reported cases. Neurology 2020; 95 (24): e3373-e3385. doi: 10.1212/ WNL.0000000000010851

[7] Siepmann T, Sedghi A, Simon E et al. Increased risk of acute stroke among patients with severe COVID-19: A multicenter study and metaanalysis. Eur J Neurol 2021; 28 (1): 238-247. 10.1111/ene.14535

[8] Siow I, Lee KS, Zhang JJY et al. Stroke as a neurological complication of COVID-19: a systematic review and meta-analysis of incidence, outcomes and predictors [published online ahead of print, 2020 Dec 15]. J Stroke Cerebrovasc Dis 2020; 30 (3): 105549. doi: 10.1016/j. jstrokecerebrovasdis.2020.105549

[9] Siepmann T, Sedghi A, Barlinn J et al. Association of history of cerebrovascular disease with severity of COVID-19 [published online ahead of print, 2020 Aug 6]. J Neurol 2020; 1-12. 10.1007/s00415-020-10121-0

[10] Kummer BR, Klang E, Stein LK et al. History of stroke is independently associated with in-hospital death in patients with COVID-19. Stroke 2020; 51 (10): 3112-3114. doi: 10.1161/STROKEAHA. 120.030685

[11] Escalard S, Chalumeau V, Escalard C et al. Early brain imaging shows increased severity of acute ischemic strokes with large vessel occlusion in COVID-19 patients. Stroke 2020; 51 (11): 3366-3370. doi: 10.1161/ STROKEAHA.120.031011
[12] Bekelis K, Missios S, Ahmad J et al. Ischemic stroke occurs less frequently in patients with COVID-19: a multicenter cross-sectional study. Stroke 2020; 51 (12): 3570-3576. doi: 10.1161/STROKEAHA.120.031217

[13] Merkler AE, Parikh NS, Mir S et al. Risk of ischemic stroke in patients with coronavirus disease 2019 (COVID-19) vs patients with influenza [published online ahead of print, 2020 Jul 2]. JAMA Neurol 2020; 77 (11): 1-7. 10.1001/jamaneurol.2020.2730

[14] Ellul MA, Benjamin L, Singh B et al. Neurological associations of COVID19. Lancet Neurol 2020; 19 (9): 767-783. doi: 10.1016/S1474-4422 (20)30221-0

[15] Ntaios G, Michel P, Georgiopoulos G et al. Characteristics and outcomes in patients with COVID-19 and acute ischemic stroke: the global COVID-19 stroke registry. Stroke. 2020; 51 (9): e254-e258. doi: 10.1161/STROKEAHA. 120.031208

[16] Yaghi S, Ishida K, Torres J et al. SARS-CoV-2 and stroke in a New York healthcare system [published correction appears in Stroke. 2020 Aug;51(8):e179]. Stroke 2020; 51 (7): 2002-2011. doi: 10.1161/ STROKEAHA. 120.030335

[17] Fifi JT, Mocco J. COVID-19 related stroke in young individuals. Lancet Neurol 2020; 19 (9): 713-715. doi: 10.1016/S1474-4422(20)30272-6

[18] Spence JD, De Freitas GR, Pettigrew LC et al. Mechanisms of stroke in COVID-19. Cerebrovasc Dis 2020; 49 (4): 451-458. doi: 10.1159/ 000509581

[19] Carod-Artal F]. Neurological complications of coronavirus and COVID19. Complicaciones neurológicas por coronavirus y COVID-19. Rev Neurol 2020; 70 (9): 311-322. doi: 10.33588/rn.7009.2020179

[20] Franceschi AM, Ahmed O, Giliberto L et al. Hemorrhagic posterior reversible encephalopathy syndrome as a manifestation of COVID-19 infection. AJNR Am J Neuroradiol 2020; 41 (7): 1173-1176. 10.3174/ajnr.A6595

[21] Lee SG, Fralick M, Sholzberg M. Coagulopathy associated with COVID19. CMAJ 2020; 192 (21): E583. doi:10.1503/cmaj.200685

[22] Cristiano A, Fortunati V, Cherubini F et al. Anti-phospholipids antibodies and immune complexes in COVID-19 patients: a putative role in disease course for anti-annexin- $\mathrm{V}$ antibodies [published online ahead of print, 2021 Jan 19]. Clin Rheumatol 2021; 1-7. 10.1007/s10067-021-05580-3

[23] Zhang Y, Xiao M, Zhang S et al. Coagulopathy and antiphospholipid antibodies in patients with Covid-19. N Engl J Med 2020; 382 (17): e38. doi: 10.1056/NEJMc2007575

[24] Hamadé A, Woehl B, Harzallah I et al. Antiphospholipid antibodies in patients with coronavirus disease 2019 infection hospitalized in conventional unit [published online ahead of print, 2020 Dec 2]. Blood Coagul Fibrinolysis 2020. doi: 10.1097/MBC.0000000000000963

[25] Van Der Linden J, Almskog L, Liliequist A et al. Thromboembolism, hypercoagulopathy, and antiphospholipid antibodies in critically ill coronavirus disease 2019 patients: a before and after study of enhanced anticoagulation. Crit Care Explor 2020; 2 (12): e0308. doi: 10.1097/ CCE.0000000000000308

[26] Iba T, Levy JH, Connors JM et al. The unique characteristics of COVID-19 coagulopathy. Crit Care 2020; 24 (1): 360

[27] Thachil J, Tang N, Gando S et al. ISTH interim guidance on recognition and management of coagulopathy in COVID-19. J Thromb Haemost 2020; 18 (5): 1023-1026. doi: 10.1111/jth. 14810

[28] Magro C, Mulvey JJ, Berlin D et al. Complement associated microvascular injury and thrombosis in the pathogenesis of severe COVID-19 infection: a report of five cases. Transl Res 2020; 220: 1-13

[29] Rapkiewicz AV, Mai X, Carsons SE et al. Megakaryocytes and platelet-fibrin thrombi characterize multi-organ thrombosis at autopsy in COVID19: a case series. EClinicalMedicine 2020; 24: 100434. Published 2020 Jun 25. doi: 10.1016/j.eclinm.2020.100434

[30] Chen W, Pan JY. Anatomical and pathological observation and analysis of SARS and COVID-19: microthrombosis is the main cause of death. Biol Proced Online 2021; 23 (1): 4 
[31] Middeldorp S, Coppens M, Van Haaps TF et al. Incidence of venous thromboembolism in hospitalized patients with COVID-19. J Thromb Haemost 2020; 18 (8): 1995-2002. doi: 10.1111/jth.14888

[32] Cui S, Chen S, Li X et al. Prevalence of venous thromboembolism in patients with severe novel coronavirus pneumonia. J Thromb Haemost 2020 Jun; 18(6): 1421-1424. doi: 10.1111/jth.14830. Epub 2020 May 6. PMID: 32271988; PMCID: PMC7262324.

[33] Hamming I, Timens W, Bulthuis ML et al. Tissue distribution of ACE2 protein, the functional receptor for SARS coronavirus. A first step in understanding SARS pathogenesis. J Pathol 2004; 203 (2): 631-637. 10.1002/path.1570

[34] Varga Z, Flammer AJ, Steiger P et al. Endothelial cell infection and endotheliitis in COVID-19. Lancet 2020; 395 (10234): 1417-1418. doi: 10.1016/S0140-6736(20)30937-5

[35] Huang C, Wang Y, Li X et al. Clinical features of patients infected with 2019 novel coronavirus in Wuhan, China. Lancet 2020; 395 (10223): 497-506. doi: 10.1016/S0140-6736(20)30183-5

[36] McGonagle D, Bridgewood C, Ramanan AV et al. COVID-19 vasculitis and novel vasculitis mimics [published online ahead of print, 2021 Jan 7]. Lancet Rheumatol 2021. doi: 10.1016/S2665-9913(20)30420-3

[37] Hernández-Fernández F, Sandoval Valencia H, Barbella-Aponte RA et al. Cerebrovascular disease in patients with COVID-19: Neuroimaging, histological and clinical description. Brain 2020; 143 (10): 3089-3103. doi: 10.1093/brain/awaa239

[38] Akhmerov A, Marbán E. COVID-19 and the heart. Circ Res 2020; 126 (10): 1443-1455

[39] Puntmann VO, Carerj ML, Wieters I et al. Outcomes of cardiovascular magnetic resonance imaging in patients recently recovered from coronavirus disease 2019 (COVID-19) [published correction appears in JAMA cardiol. 2020 Nov 1;5(11):1308]. JAMA Cardiol 2020; 5 (11): 12651273. doi: $10.1001 /$ jamacardio.2020.3557

[40] Madjid M, Safavi-Naeini P, Solomon SD et al. Potential effects of coronaviruses on the cardiovascular system: a review. JAMA Cardiol 2020; 5 (7): 831-840. doi: 10.1001/jamacardio.2020.1286

[41] Hoyer C, Ebert A, Huttner HB et al. Acute stroke in times of the COVID19 pandemic: a multicenter study. Stroke 2020; 51 (7): 2224-2227. 10.1161/STROKEAHA. 120.030395

[42] Kerleroux B, Fabacher T, Bricout $\mathrm{N}$ et al. Mechanical thrombectomy for acute ischemic stroke amid the COVID-19 outbreak: decreased activity, and increased care delays. Stroke 2020; 51 (7): 2012-2017. doi: 10.1161/STROKEAHA.120.030373

[43] Rudilosso S, Laredo C, Vera V et al. Acute stroke care is at risk in the era of COVID-19: experience at a comprehensive stroke center in Barcelona. Stroke 2020; 51 (7): 1991-1995. doi: 10.1161/ STROKEAHA.120.030329

[44] Abootalebi S, Aertker BM, Andalibi MS et al. Call to action: SARS-CoV-2 and CerebrovAscular DisordErs (CASCADE). J Stroke Cerebrovasc Dis 2020; 29 (9): 104938. doi: 10.1016/j.jstrokecerebrovasdis.2020.104938
[45] Goyal M, Ospel JM, Southerland AM et al. Prehospital triage of acute stroke patients during the COVID-19 pandemic. Stroke 2020; 51 (7): 2263-2267. doi: 10.1161/STROKEAHA.120.030340

[46] Khosravani H, Rajendram P, Notario L et al. Protected code stroke: hyperacute stroke management during the coronavirus disease 2019 (COVID-19) pandemic. Stroke 2020; 51 (6): 1891-1895. doi: 10.1161/ STROKEAHA. 120.029838

[47] Tiedt S, Bode FJ, Uphaus T et al. Impact of the COVID-19-pandemic on thrombectomy services in Germany. Neurol Res Pract 2020; 2:44.Published 2020 Nov 23. doi: 10.1186/s42466-020-00090-0

[48] Hajdu SD, Pittet V, Puccinelli F et al. Acute stroke management during the COVID-19 pandemic: does confinement impact eligibility for endovascular therapy? Stroke 2020; 51 (8): 2593-2596. doi: 10.1161/ STROKEAHA. 120.030794

[49] Uphaus T, Gröschel S, Hayani E et al. Stroke care within the COVID-19 pandemic-increasing awareness of transient and mild stroke symptoms needed. Front Neurol 2020; 11: 581394. doi: 10.3389/fneur.2020.581394

[50] Barlinn K, Siepmann T, Pallesen LP et al. Universal laboratory testing for SARS-CoV-2 in hyperacute stroke during the COVID-19 pandemic. J Stroke Cerebrovasc Dis 2020 Sep; 29(9): 105061. doi: 10.1016/j. jstrokecerebrovasdis.2020.105061

[51] Baracchini C, Pieroni A. Acute stroke treatment during coronavirus disease 2019 pandemic. Curr Opin Neurol 2021; 34 (1): 11-17. doi: 10.1097/WCO.0000000000000886

[52] Friedlich D, Newman T, Bricker S. Impact of the COVID-19 pandemic on stroke epidemiology and clinical stroke practice in the US [published online ahead of print, 2021 Jan 26]. J Stroke Cerebrovasc Dis 2021; 30 (4): 105639. doi: 10.1016/j.jstrokecerebrovasdis.2021.105639

[53] Guan W], Ni ZY, Hu Y et al. Clinical characteristics of coronavirus disease 2019 in China. N Engl J Med 2020; 382 (18): 1708-1720. doi: 10.1056/ NEJMoa2002032

[54] MacDougall K, Spyropoulos AC. Prevention of venous thromboembolism in acutely ill medical patients: a new era [published online ahead of print, 2021 Feb 6]. Semin Respir Crit Care Med 2021. doi: 10.1055/s0041-1723018

[55] Tiwari NR, Khatib KI, Dixit SB et al. Anticoagulation in COVID - 19: an update. J Crit Care Med (Targu Mures) 2020; 6 (4): 217-223. 10.2478/ jccm-2020-0033

[56] Lemos ACB, Do Espírito Santo DA, Salvetti MC et al. Therapeutic versus prophylactic anticoagulation for severe COVID-19: a randomized phase II clinical trial (HESACOVID). Thromb Res 2020 Dec;196:359-366. doi: 10.1016/j.thromres.2020.09.026. Epub 2020 Sep 21. PMID: 32977137; PMCID: PMC7503069.

[57] https://www.nih.gov/news-events/news-releases/nih-activ-trial-blood-t hinners-pauses-enrollment-critically-ill-covid-19-patients

[58] Berlit P, Bösel J, Gahn G et al. "Neurological manifestations of COVID19 " - guideline of the German society of neurology. Neurol Res Pract 2020; 2: 51. Published 2020 Dec 2. doi: 10.1186/s42466-020-00097-7 